\title{
TOXICIDADE PARA LAGARTAS DE Phthorimaea operculella (Zell.) DOS ALELOQUÍMICOS 2-TRIDECANONA E 2-UNDECANONA PRESENTES EM TOMATEIRO (Lycopersicon spp.)
}

\author{
M.U. VENTURA'; J.D. VENDRAMIM \\ 1 Depto. de Agronomia-UEL, C.P. 6001, CEP: 86051-970, Londrina, PR. \\ ${ }^{2}$ Depto. de Entomologia-ESALQ/USP, C.P. 9, CEP: 13418-900, Pinacicaba, SP.
}

RESUMO: Avalion-se a toxicidade para Phthorimaea operculella (Zell.) dos aleloquímicos 2-tridecanona e 2undecanona, presentes nos exsudatos glandulares dos tricomas de Lycopersicon spp. Determinou-se a toxicidade dos exsudatos de $L$. hirsutum f. glabratum PI 134417 (DL ${ }_{s 0}$ ), do 2-tridecanona (dose letal: DL $_{s 0}$ e concentração letal: $\mathrm{CL}_{30}$ ) e do 2-undecanona $\left(\mathrm{CL}_{30}\right.$ ) para lagartas de primeiro instar desse inseto. $A \mathrm{DL}_{30}$ foi de 5,54 glandulas e 775,53 ng por lagarta, respectivamente. Em relação à concentração letal, o 2-tridecanona foi mais tóxico do que o 2undecanona. A toxicidade $\left(\mathrm{CL}_{50}\right)$ do 2-tridecanona aumentou com a elevaçáo da temperatura de 27 para $32^{\circ} \mathrm{C}$ e para o 2-undecanona ocorreu o inverso. Concluiu-se que estes compostos podem ter grande importância na resisténcia do tomateiro para esta praga.

Descritores: Insecta, tomateiro, praga, resistência de plantas

\section{TOXICITY OF 2-TRIDECANONE AND 2-UNDECANONE ALLELOCHEMICALS PRESENT IN TOMATO (Lycopersicon spp.) FOR Phthorimaea operculella (Zell.) LARVAE}

ABSTRACT: This research deals with the toxicity of 2-tridecanone and 2-undecanone allelochemicals, present in the grandular trichomes of Lycopersicon spp., to Phthorimaea operculella (Zell.). The toxicity of the exsudates of L. hirsutum f. glabratum PI 134117 ( $\left(D_{s 0}\right)$, of the 2-tridecanone (lethal dose: $L D_{s 0}$ and lethal concentration: $L C_{s 0}$ ) and 2 -undecanone $\left(\mathrm{LC}_{30}\right.$ ) to first instar larvae of this pest were determined. The $\mathrm{LD}_{50}$ value was 5.54 glands and 775.53 ng per larvae, respectively. With respect to the lethal concentration, the 2-tridecanone was more toxic than the 2-undecanone. The toxicity $\left(\mathrm{CL}_{5_{0}}\right)$ of the 2-tridecanone increased with the elevation of the temperature from 27 to $32^{\circ} \mathrm{C}$, but decreased with the 2-undecanone. It was concluded that these compounds can have great importance in the resistance of the tomato plant to this pest.

Key Words: Insecta, tomato, pest, plant resistance

\section{INTRODUÇÃO}

Os aleloquímicos 2-tridecanona e 2undecanona são metil-cetonas presentes nos exsudatos glandulares dos tricomas do tipo VI de Lycopersicon spp. A visualização desses tricomas pode ser feita facilmente utilizando-se o microscópio esteroscópico, pois são os mais conspícuos dos diversos tipos que estão presentes em folhas e frutos de tomateiro.

A extremidade apical desses tricomas é multicelular e apresenta forma circular, diferindo, no entanto, na sua morfologia, em função da espécie de tomateiro. Enquanto nos materiais comerciais (L. esculentum) a divisão entre as células é bastante pronunciada o que leva à formação de quatro lóbulos, no tomateiro selvagem, $\boldsymbol{L}$. hirsutum, esta divisão é muito pouco evidente, resultando na formação de apenas um lóbulo (SNYDER \& CARTER, 1985).

WILLIAMS et al. (1980) isolaram e identificaram o 2-tridecanona da espécie selvagem L. hirsutum f. glabratum PI 134417. Verificaram que, nesta espécie, esta substância foi encontrada em concentração 75 vezes maior do que em $L$. esculentum. Também constataram que este composto apresentou-se tóxico para Manduca sexta L., Helicoverpa zea Bod. e Aphis gossypii Glover, quando os insetos foram colocados sobre papelfiltro tratado com esta substância.

Vários autores comprovaram a importância destes compostos na resistência de $\mathrm{L}$. hirsutum $\mathrm{f}$. glabratum a pragas, como por exemplo para $H$. zea (DIMOCK et al., 1982); $M$. sexta (SCHWARTZ \& SNYDER, 1983); Leptinotarsa decemlineata (Say) (KENNEDY \& SORENSON, 1985); Keiferia 
bycopersicella (Wals.) e Spodoptera exigua (Hub.) (LIN et al., 1987) e Scrobipalpuloides absoluta (Meyrick) (GIUSTOLIN, 1991).

O objetivo do presente trabalho foi estudar a toxicidade dos aleloquímicos 2-tridecanona e 2undecanona, bem como dos exsudatos grandulares de L. hirsutum f. glabratum PI 134417 para lagartas de primeiro instar de Phthorimaea operculella (Zell.), visando avaliar o potencial desses aleloquímicos cómo fator de resisténcia a essa praga em tomateiro.

\section{MATERIAL E MÉTODOS}

0 presente trabalho foi desenvolvido no laboratório de Resistência de Plantas a Insetos do Departamento de Entomologia da Escola Superior de Agricultura "Luiz de Queiroz", ESALQ/USP, em Piracicaba, SP. A criação de P. operculella também foi realizada neste laboratório. Os insetos foram mantidos à temperatura de $25 \pm 1^{\circ} \mathrm{C}$. Quando os ovos estavam escuros, próximos ao momento da eclosão das lagartas, foram colocados sobre tubérculos de batata perfurados com pinça para facilitar a entrada dos insetos. As lagartas alimentaram-se dos tubérculos durante todo o período larval. A bandeja plástica que continha os tuberculos foi forrada com areia de rio autoclavada para servir de substrato na fase de pupa. A cada dois dias a areia foi peneirada para retirada das pupas. Em seguida, as pupas foram sexadas e transferidas em grupos de cinco casais para gaiolas feitas de tubo de PVC de $10 \mathrm{~cm}$ de diâmetro e 20 cm de comprimento. A extremidade superior do tubo foi fechada com um pedaço de tecido (fil6) de malha fina, afixado com elástico. Sobre o tecido foi colocado papel-filtro onde os insetos fizeram a postura.

\section{Toxicidade dos pelos glandulares de $L$. hirsutum f. glabratum PI 134417}

Esta avaliação foi feita com base na metodologia utilizada por LIN et al. (1987) para Keiferia lycopersicella (Wals.) e Spodoptera exigua (Hub.). Esta metodologia consiste em se colocar diretamente a lagarta recém-eclodida em contacto com o exsudato dos pélos de folhas retiradas da planta. Quando as lagartas são expostas aos tricomas, a glândula rompe-se ficando aderida ao corpo desta. As folhas utilizadas (terceira a partir do ponteiro) foram destacadas de plantas (cultivadas em casa-de-vegetação) com 45 dias de idade. Foram utilizadas as doses de 2, 4, 6, 8 e 10 glândulas por inseto, além de uma testemunha. Estas doses foram estabelecidas em testes preliminares. Para cada dose foram tratados 60 insetos. Após o contacto, observou-se o comportamento das lagartas por 5 minutos. A seguir, foram transferidas para placas de Petri de $5,5 \mathrm{~cm}$ de diâmetro vedadas com filme de PVC transparente e mantidas, sem alimento, numa câmara incubadora do tipo B.O.D. regulada a $26^{\circ} \mathrm{C}$, no escuro. Ap6s 24 horas foi feita a avaliação do número de insetos mortos. Era considerada morta a lagarta que não apresentava movimentos após ter sido tocada trés vezes por um pincel fino.

\section{Toxicidade do 2-tridecanona e 2-undecanona}

Determinacĩo da dose letal (DL $\left.{ }_{s}\right)$ : A metodologia utilizada tambem foi proposta por LIN et al. (1987). Foram feitas aplicações tópicas de 2-tridecanona comercial ( $99 \%$, Aldrich ${ }^{\circ}$ ), dissolvido em isooctano, em lagartas recém eclodidas, utilizando uma seringa de Hamilton de $10 \mu \mathrm{l}$. Foram aplicados $0,05 \mu \mathrm{l}$ de solução por lagarta. As doses utilizadas foram $300,400,500,600,700,800$ e $1000 \mathrm{ng} /$ lagarta, alem de uma testemunha $(0,05$ $\mu l$ de isooctano/lagarta). Foram usadas 60 lagartas por tratamento. Os insetos foram mantidos sem alimento em placas de Petri de $5,5 \mathrm{~cm}$ de diâmetro vedadas com filme de PVC transparente. As placas foram colocadas em B.O.D. regulada à $26^{\circ} \mathrm{C}$, no escuro. Vinte e quatro horas após a aplicação, foram feitas as avaliaçōes de mortalidade, conforme critério descrito no teste com exsudatos. Não foi avaliada a toxicidade dérmica do 2-undecanona, por este composto ser muito volátil e estar, na planta, em concentrações bem inferiores ao 2tridecanona (LIN et al., 1987).

Determinaçũo da concentragão letal ( $\mathrm{CL}_{\mathrm{sa}}$ ): Para esta determinação, que foi feita com base na metodologia de DIMOCK et al. (1982), lagartas recém eclodidas foram colocadas em placas de Petri de $8,5 \mathrm{~cm}$ de diâmetro com papel-filtro tratado com 2-tridecanona ou 2-undecanona (ambos produtos comerciais, $99 \%$, Aldrich ${ }^{\circ}$ ). Para evitar a volatilização dos aleloquímicos as placas foram vedadas com filme de PVC transparente. Os aleloquímicos, testados isoladamente, foram previamente diluídos em clorofórmio para então serem pipetados sobre o papel-filtro, aguardandose alguns minutos (necessários para evaporação do cloroformio) antes de se colocarem as lagartas. As doses utilizadas foram $3,6,9,12$ e $15 \mu \mathrm{g}$ de 2 tridecanona e $15,30,45,60$ e $75 \mu \mathrm{g}$ de 2- 
undecanona por $\mathrm{cm}^{2}$ de papel-filtro, além da testemunha (clorofórmio). Foram realizados testes à $27^{\circ} \mathrm{C}$ e d $32^{\circ} \mathrm{C}$, para se estimar o efeito da variação da temperatura na toxicidade desse aleloquímico para $P$. operculella. Os insetos (60 por tratamento) permaneceram nas placas em B.O.D. durante seis horas, no escuro e sem alimento. Após este periodo, foi contado o número de insetos mortos, conforme critério descrito no teste com exsudatos. A análise de próbites foi feita em microcomputador, utilizando programa desenvolvido no Departamento de Entomologia da ESALQ/USP (HADDAD, 1986).

\section{RESULTADOS E DISCUSSÃO}

Toxicidade dos pellos glandulares de $L$. hirsutum f. glabratum PI 134417

A dose letal dos exsudatos glandulares de L. hirsutum f. glabratum PI 134417 para 50\% da população foi de 5,54 exsudatos por lagarta (TABELA 1).

Em comparação com os resultados obtidos por LIN et al. (1987), verifica-se que P. operculella É bem mais tolerante à exposição aos exsudatos do que Keiferia bycopersicella (Wals.), para a qual apenas um pêlo glandular por lagarta provoca mortalidade superior a $50 \%$. Já em comparação a Spodoptera exigua (Hub.), P. operculella foi bem mais sensível. A variação na toxicidade desses exsudatos pode ser devido, ao menos em parte, ds diferenças de tamanho desses insetos, já que, $\boldsymbol{K}$. hycopersicella $\in$ menor que $P$. operculella e $S$. exigua é maior.

Foi observado também que o efeito do exsudato é principalmente químico e não mecânico. Após o contacto com o corpo da lagarta, a glândula se rompe e a lagarta inicialmente se contorce, mas apбs alguns segundos consegue se locomover, não ficando presa no pêlo glandular. Cessa seus movimentos somente se o número de pêlos glandulares for suficiente para matá-la.

Toxicidade do 2-tridecanona e 2-undecanona para lagartas de primeiro fnstar de $P$. operculella

Dose letal ( $\left.\mathrm{DL}_{s 0}\right)$ : $\mathrm{O}$ valor obtido para a $\mathrm{DL}_{s 0}$ para lagartas de primeiro ínstar de $P$. operculella $(775,53$ ng/lagarta, TABELA 1) foi superior ao estabelecido por LIN et al. (1987) para $K$. hycopersicella (51,00 ng/lagarta). Entretanto, foi inferior ao valor obtido por estes mesmos autores para $S$. exigua (3440 ng/ lagarta). Esse resultado justifica a menor toxicidade dos exsudatos glandulares para $P$. operculella em relação a $K$. bycopersicella, bem como a maior toxicidade quando a comparação é feita com $S$. exigua.

Concentraçăo letal $\left(\mathrm{CL}_{s o}\right)$ : A toxicidade do 2tridecanona para lagartas de $P$. operculella, expressa em concentração letal ( $\mu \mathrm{g}$ de produto por $\mathrm{cm}^{2}$ de papel-filtro), foi bastante superior à constatada para - 2-undecanona, tanto à $27^{\circ} \mathrm{C}(8,63$ e $38,76 \mu \mathrm{g} /$ $\mathrm{cm}^{2}$, respectivamente), como à $32^{\circ} \mathrm{C}(5,19$ e 45,48 $\mu \mathrm{g} / \mathrm{cm}^{2}$, respectivamente) (TABELA 1 ).

Essa maior toxicidade do 2-tridecanona também foi observada por DIMOCK et al. (1982) para Helicoverpa zea (Bod.), que obtiveram a concentração letal de $17,05 \mu \mathrm{g} / \mathrm{cm}^{2}$ para o 2tridecanona e $64,19 \mu \mathrm{g} / \mathrm{cm}^{2}$ para o 2-undecanona. Observa-se que os valores obtidos para $\boldsymbol{H}$. zea por estes autores foram maiores do que os observados para $P$. operculella no presente trabalho, caracterizando-se, dessa forma, uma maior toxicidade desses compostos para lagartas de $P$. operculella do que para $H$. zea. Já para Leptinotarsa decemlineata (Say), KENNEDY \& SORENSON (1985) obtiveram $\mathrm{CL}_{50}$ de $26,94 \mu \mathrm{g} / \mathrm{cm}^{2}$, valor também maior do que o conseguido para $P$. operculella.

TABELA 1. Toxicidade ( $\left(\mathrm{LL}_{50}\right.$ ) dos pêlos glandulares de Lycopersicon hirsutum f. glabratum $\mathrm{Pl} 134417$ e do 2-tridecanona para lagartas de primeiro instar de Phthorimaea operculella, mantidas no escuro, 24 horas após a aplicação. Temp. $26^{\circ} \mathrm{C}$; UR $70 \pm 10 \%$.

\begin{tabular}{llc}
\hline \hline PARÂMETRO & $\mathrm{DL}_{s 0}$ & $($ (I.C.) \\
\hline Pêlos glandulares (glândulas/lagarta) & 5,54 & $(4,31-7,13)$ \\
2-tridecanona (ng/lagarta) & 775,53 & $(667,59-855,06)$ \\
\hline
\end{tabular}

'Intervalo de confiança ao nível de $95 \%$ de probabilidade 
TABELA 2 - Toxicidade $\left(\mathrm{CL}_{s 0}\right)$ de 2-tridecanona e 2-undecanona, aplicados sobre papel filtro, seis horas após o contacto com lagartas de primeiro instar de Phthotimaea operculella, mantidas no escuro em duas temperaturas (UR: $70 \pm 10 \%$ ).

\begin{tabular}{lcc}
\hline \hline & $\mathrm{CL}_{50}\left(\mathrm{gg} / \mathrm{cm}^{2}\right.$ de papel-filtro & $(\mathrm{I} . \mathrm{C})^{\mathrm{t}}$ \\
\hline ALELOQUIMICOS & $27^{\circ} \mathrm{C}$ & $32^{\circ} \mathrm{C}$ \\
2-tridecanona & $8,63(6,29-11,84)$ & $5,19(3,34-8,07)$ \\
2-undecanona & $38,76(28,06-53,33)$ & $45,48(25,81-80,12)$ \\
\hline
\end{tabular}

'Intervalo de confiança ao nível de $95 \%$ de probabilidade.

Em relação às temperaturas, observou-se a toxicidade diferenciada dos dois aleloquímicos. Enquanto que para o 2-tridecanona houve aumento na toxicidade com a elevação da temperatura de 27 para $32^{\circ} \mathrm{C}$, com o 2-undecanona ocorreu o inverso. A diminuição da toxicidade do 2-undecanona com o aumento da temperatura pode estar relacionada com a alta volatilização deste composto que, segundo LIN et al. (1987), é bem superior à apresentada pelo 2-tridecanona. Com essa alta volatilização, o produto teria se concentrado na parte superior da placa, diminuindo o contacto da substância com as lagartas, já que, conforme foi observado, as lagartas tendiam a se locomover para a parte inferior da placa, mantendo-se sob o papelfiltro. A variação na toxicidade desse aleloquímico em função da temperatura permite inferir que na planta o grau de resistência mediado por estes compostos é influenciado pelas condições de temperatura ambiente, o que já foi citado em vários casos de resistência de plantas a insetos (LARA, 1991).

Com base nos resultados obtidos, pode-se observar que os exsudatos glandulares de $L$. hirsutum f. glabratum PI 134417 e o 2-tridecanona, em aplicação tópica, são tóxicos para as lagartas, sendo necessários 5,54 exsudatos ou 775,35 ng por lagarta de primeiro ínstar, respectivamente, para provocar a mortalidade de $50 \%$ da população. Em testes de concentração letal, o 2-tridecanona apresenta maior toxicidade do que o 2-undecanona e a elevação da temperatura, de 27 para $32^{\circ} \mathrm{C}$, aumenta a toxicidade $\left(\mathrm{CL}_{30}\right)$ do 2-tridecanona e reduz a do 2-undecanona.

Conclui-se que estes aleloquímicos são condicionadores de resistência, principalmente o 2-tridecanona. Porem, existe a necessidade de se pesquisar a existência ou não de outros fatores.

\section{REFERÊNCIAS BIBLIOGRÁFICAS}

DIMOCK, M.B.; KENNEDY, G.G.; WILLIAMS, W.G. Toxicity of analogs of 2-tridecanone, a naturally occurring toxicant from a wild tomato. Jourmal of Chemical Ecology, v.8, n.5, p.837-42, 1982.

GIUSTOLIN, T.A. Efeito dos aleloquímicos 2tridecanona e 2-undecanona, presentes em Lycopersicon spp., sobre a biologia da traça do tomateiro Scrobipalpuloides absoluta (Meyrick, 1917) (Lep., Gelechiidae). Piracicaba, 1991. 155p. Dissertação (Mestrado) - Escola Superior de Agricultura "Luiz de Queiroz", Universidade de Sז̃o Paulo.

HADDAD, M.L. Análise de próbites. In: ALVES, S.B., ed. Controle microbiano de insetos. São Paulo: Manole, 1986. p.374-83.

KENNEDY, G.G.; SORENSON, C.F. Role of glandular trichomes in the resistance of Lycopersicon hirsutum f. glabratum to Colorado potato beetle (Coleoptera: Chrysomelidae). Journal of Economic Entomology, v.78, p.547-51, 1985.

LARA, F.M. Princípios de resistência de plantas a insetos. 2.ed. São Paulo: Icone, 1991. 336p.

LIN, S.Y.H.; TRUMBLE, J.T.; KUMAMOTO, J. Activity of volatile compounds in glandular trichomes of Lycopersicon species against two insect herbivores. Journal of Chemical Ecology, v.13, n.4, p.837-50, 1987.

SCHWARTZ, R.F.; SNYDER, J.C. Characterization of resistance to tobacco hormworn in Lycopersicon leaflets. Hort Science, v.18, n.2, p.170, 1983.

SNYDER, J.C.; CARTER, C.D. Trichomes on leaves of Lycopersicon hirsutum, $L$. esculentum and their hybrids. Euphytica, v.34, p.53-64, 1985.

WILLIAMS, W.G.; KENNEDY, G.G.; YAMAMOTO, R.T.; THACKER, J.D.; BORDNER, J. 2-tridecanone: a naturally occurring inseticide from the wild tomato Lycopersicon hirsutum f. glabranum. Science, v.207, p.888-9, 1980.

Recebido para publicação em 09.01.95

Aceito para publicação em 04.05.95 